\title{
Solitons, Shock Waves and Conservation Laws of Rosenau-KdV-RLW Equation with Power Law Nonlinearity
}

\author{
Polina Razborova ${ }^{1}$, Bouthina Ahmed ${ }^{2}$ and Anjan Biswas ${ }^{1,3, *}$ \\ ${ }^{1}$ Department of Mathematical Sciences, Delaware State University, Dover, DE 19901-2277, USA \\ 2 Department of Mathematics, Girl's College, Ain Shams University, Cairo-11757, Egypt \\ ${ }^{3}$ Department of Mathematics, Faculty of Sciences, King Abdulaziz University, Jeddah-21589, Saudi Arabia
}

Received: 9 Mar. 2013, Revised: 10 Jul. 2013, Accepted: 12 Jul. 2013

Published online: 1 Mar. 2014

\begin{abstract}
This paper obtains solitary waves, shock waves and singular solitons alon with conservation laws of the Rosenau Kortewegde Vries regularized long wave (R-KdV-RLW) equation with power law nonlinearity that models the dynamics of shallow water waves. The ansatz approach and the semi-inverse variational principle are used to obtain these solutions. The constraint conditions for the existence of solitons are also listed.
\end{abstract}

Keywords: solitons; integrability; conservation laws

\section{Introduction}

The theory of solitons is one of the most progressive research areas in applied mathematics and theoretical physics [1-20]. Solitons or nonlinear waves appear everywhere in daily lives. Optical solitons are the most fundamental pulses that travel through the optical fibers for transcontinental and transoceanic distances. The modern telecommunication system in information sciences has advanced because of the progress in the research on optical solitons [9, 12]. Apart from nonlinear optics, solitons are also observed on lake shores, beaches as well as canals. The dynamics of dispersive shallow water waves is extensively studied by various known models. These are the Rosenau-Kawahara equation, Rosenau-KdV equation, Rosenau-RLW equation and many others. This paper is going to introduce the R-KdV-RLW equation, with power law nonlinearity, that serves as yet another model to study the shallow water waves.

The aim of this paper is to solve the R-KdV-RLW equation by the ansatz method and the semi-inverse variational principle (SVP). The solitary waves, shock waves and the singular solitons will be determined. It will be seen that the shock wave solutions will be available for only two particular values of the power law nonlinearity parameter. However, the solitary waves and the singular solitons are defined as long as the power law nonlinearity parameter is bigger than unity.

\section{Governing Equation}

The dimensionless form of the R-KdV-RLW equation that is going to be studied in this paper is given by $[5,13,14,20]$

$$
q_{t}+a q_{x}+b_{1} q_{x x x}+b_{2} q_{x x t}+c q_{x x x x}+k\left(q^{n}\right)_{x}=0
$$

Here, $q(x, t)$ is the nonlinear wave profile where $x$ and $t$ are the spatial and temporal variables respectively. The first term is the linear evolution term, while the coefficient of $a$ is the advection or drifting term. The two dispersion terms are the coefficients of $b_{j}$ for $j=1,2$. The higher order dispersion term is the coefficient of $c$ while the coefficient of nonlinearity is $k$ where $n$ is the nonlinearity parameter. These are all known and given parameters. It is, however, necessary to note that

$$
n>1
$$

in order for the solitons to exist.

\footnotetext{
* Corresponding author e-mail: biswas.anjan@gmail.com
} 
Equation (1) is a combination of R-KdV equation [13] and R-RLW equation both of which are studied in details in the context of shallow water waves. This R-KdV-RLW equation is a combination of the two forms of dispersive shallow water waves that is analogue to the improved $\mathrm{KdV}$ equation. Therefore this equation models dispersive shallow water waves with the equal-width factor taken into account.

Equation (1) will be solved by the ansatz method. The traveling wave hypothesis fails to retrieve as wave of permanent form for equation (1). The ansatz method will be applied to extract the solitary waves, shock waves as well as the singular solitons for the R-KdV-RLW equation (1). This study will now be detailed in the next three subsections.

\subsection{Solitary Waves}

In order to obtain the solitary wave solution to the R-KdVRLW equation, the starting hypothesis is taken to be [15]

$$
q(x, t)=A \operatorname{sech}^{p}[B(x-v t)]=A \operatorname{sech}^{p} \tau
$$

where $A$ is the amplitude of the soliton, while $B$ is the inverse width and $v$ is the soliton velocity and

$$
\tau=B(x-v t) .
$$

The value of the unknown exponent $p>0$ will be obtained by the balancing principle. Substituting equation (3) into equation (1) gives

$$
\begin{gathered}
\left\{v\left(1+b_{2} p^{2} B^{2}+c p^{4} B^{4}\right)-a-b_{1} p^{2} B^{2}\right\} \operatorname{sech}^{p} \tau \\
+B^{2}(p+1)(p+2)\left\{b_{1}-b_{2} v-2 c v B^{2}\left(p^{2}+2 p+2\right)\right\} \operatorname{sech}^{p+2} \tau \\
+c v B^{4}(p+1)(p+2)(p+3)(p+4) \operatorname{sech}^{p+4} \tau \\
-n k A^{n-1} \operatorname{sech}^{n p} \tau=0
\end{gathered}
$$

By the balancing principle, equating the exponents $n p$ and $p+4$ leads to

$$
p=\frac{4}{n-1} \text {. }
$$

Also, from equation (5), setting the coefficients of the linearly independent functions $\operatorname{sech}^{p+j} \tau$ for $j=0,2,4$ leads to

$$
A=\left[\frac{8(n+1)(n+3)(3 n+1) b_{1} c B^{4}}{k(n-1)^{2}\left\{(n-1)^{2} b_{2}+4\left(n^{2}+2 n+5\right) c B^{2}\right\}}\right]^{\frac{1}{n-1}}
$$

and the velocity $(v)$ of the soliton is

$$
v=\frac{a(n-1)^{4}+16(n-1)^{2} b_{1} B^{2}}{(n-1)^{4}+16(n-1)^{2} b_{2} B^{2}+256 c B^{4}}
$$

or

$$
v=\frac{b_{1}(n-1)^{2}}{(n-1)^{2} b_{2}+4 c B^{2}\left(n^{2}+2 n+5\right)} .
$$

Upon equating the values of the velocity of the soliton from equations (8) and (9) leads to the width of the soliton being given by

$$
B=\frac{n-1}{n+1}\left[\frac{D-\left(n^{2}+2 n+5\right) a c}{32 b_{1} c}\right]^{\frac{1}{2}},
$$

where $D$ is given by

$$
D=\sqrt{a^{2} c^{2}\left(n^{2}+2 n+5\right)^{2}+16(n+1)^{2} b_{1} c\left(b_{1}-a b_{2}\right)} .
$$

Substituting (10) into (7) leads to

$$
\begin{gathered}
A=\left[\frac{\left\{D-\left(n^{2}+2 n+5\right) a c\right\}^{2}}{8(n+1)^{2} b_{1} b_{2}+\left(n^{2}+2 n+5\right)\left\{D-\left(n^{2}+2 n+5\right) a c\right\}}\right]^{\frac{1}{n-1}} \\
\times\left[\frac{(n+3)(3 n+1)}{16(n+1) c k}\right]^{\frac{1}{n-1}},
\end{gathered}
$$

which is the amplitude of the soliton expressed completely in terms of known parameters. The width of the soliton given by equation (10), which is also in terms of known parameters, will exist provided

$$
b_{1} c\left\{D-\left(n^{2}+2 n+5\right) a c\right\}>0
$$

and equation (11) stays valid for

$$
a^{2} c^{2}\left(n^{2}+2 n+5\right)^{2}+16(n+1)^{2} b_{1} c\left(b_{1}-a b_{2}\right)>0 .
$$

Finally, the solitary wave solution to the R-KdV-RLW equation is given by

$$
q(x, t)=A \operatorname{sech}^{\frac{4}{n-1}}[B(x-v t)] .
$$

The following figure shows the profile of a solitary wave for $a=1, b_{1}=b_{2}=-1, c=k=1, v=0.5, n=2$.

\subsection{Shock Waves}

In order to extract the shock wave solution to the R-KdVRLW equation (1), the starting hypothesis is [5]

$$
q(x, t)=A \tanh ^{p} \tau
$$

for $p>0$. The definition of $\tau$ stays the same as in (4). For this case, the parameters $A$ and $B$ are free parameters while $v$ is the velocity of the shock wave. Substituting (16) into 


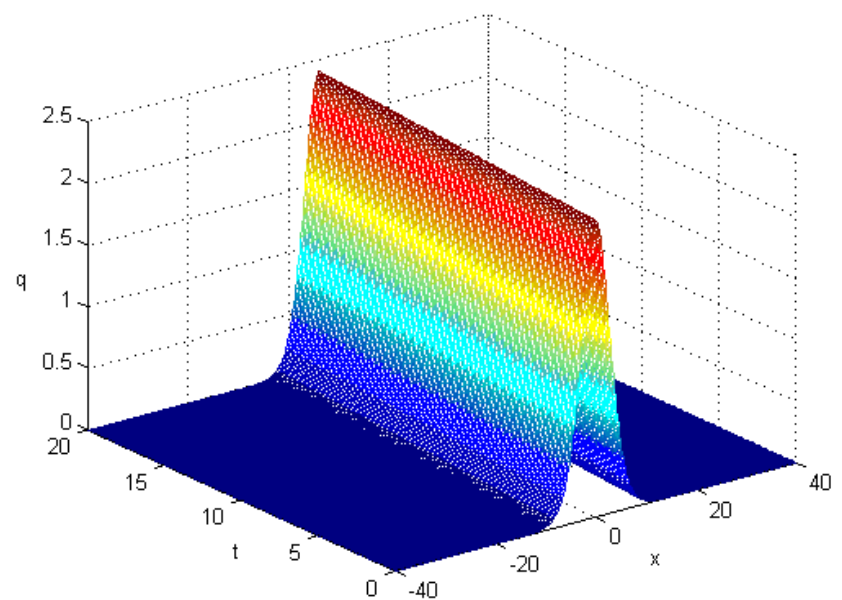

Figure 1: Soliton solution with $n=2, a=1, b 1=-1, b 2=-1, c=1, k=1, v=0.5$

(1) leads to

$$
\begin{gathered}
{\left[a-v-\left(b_{1}-b_{2} v\right)\left(3 p^{2}-3 p+2\right) B^{2}\right.} \\
\left.-2\left(5 p^{4}-10 p^{3}+25 p^{2}-20 p+8\right) c v B^{4}\right] \tanh ^{p-1} \tau \\
-\left[a-v-\left(b_{1}-b_{2} v\right)\left(3 p^{2}+3 p+2\right) B^{2}\right. \\
\left.-2\left(5 p^{4}+10 p^{3}+25 p^{2}+20 p+8\right) c v B^{4}\right] \tanh ^{p+1} \tau \\
+(p-1)(p-2) B^{2}\left\{b_{1}-b_{2} v+5\left(p^{2}-3 p+4\right) v c B^{2}\right\} \tanh ^{p-3} \tau \\
-(p+1)(p+2) B^{2}\left\{b_{1}-b_{2} v+5\left(p^{2}+3 p+4\right) v c B^{2}\right\} \tanh ^{p+3} \tau \\
-(p-1)(p-2)(p-3)(p-4) c v B^{4} \tanh ^{p-5} \tau \\
+(p+1)(p+2)(p+3)(p+4) c v B^{4} \tanh ^{p+5} \tau \\
+n k A^{n-1}\left(\tanh ^{n p-1} \tau-\tanh ^{n p+1} \tau\right)=0 \quad(17)
\end{gathered}
$$

By the balancing principle, equating the exponent pairs $(n p+1, p+5)$ or $(n p-1, p+3)$ leads to the same value of $p$ as in equation (6). Next, setting the coefficient of the linearly independent function $\tanh ^{p-5} \tau$ and $\tanh ^{p-3} \tau$ to zero leads to

$$
p=1 \text { or } p=2 \text {. }
$$

This leads to the following two sub cases that will be individually handled in the following two subsections.

\subsubsection{Case-I: $\mathrm{p}=1$}

Since $p=1$, equation (6) implies

$$
n=5 \text {. }
$$

This implies the R-KdV-RLW equation, with power law nonlinearity, transforms to

$$
q_{t}+a q_{x}+b_{1} q_{x x x}+b_{2} q_{x x t}+c q_{x x x x t}+k\left(q^{5}\right)_{x}=0 .
$$

Now, equating the coefficients of the remaining linearly independent functions $\tanh ^{p-j} \tau$, for $j=-1,1$ to zero, gives the speed of the shock wave as

$$
v=\frac{a-2 b_{1} B^{2}}{16 c B^{4}-2 b_{2} B^{2}+1}
$$

and

$$
v=\frac{a-8 b_{1} B^{2}}{136 c B^{4}-8 b_{2} B^{2}+1} .
$$

Then, equating the two values of the speed from equations (21) and (22) gives the biquadratic equation for the free parameter $B$ as

$$
24 b_{1} c B^{4}-20 a c B^{2}-\left(b_{1}-a b_{2}\right)=0,
$$

whose solution is

$$
B=\left[\frac{5 a c-\sqrt{25 a^{2} c^{2}+6 b_{1} c\left(b_{1}-a b_{2}\right)}}{12 b_{1} c}\right]^{\frac{1}{2}} .
$$

Next setting the coefficients of the linearly independent functions $\tanh ^{p+j} \tau$, for $j=3,5$ to zero leads to the values of the second free parameter $A$ as

$$
A=B\left(\frac{24 v c}{k}\right)^{\frac{1}{4}}
$$

and

$$
A=\left[\frac{6 B^{2}\left(b_{1}-b_{2} v+40 v c B^{2}\right)}{5 k}\right]^{\frac{1}{4}} .
$$

Equating the two values of the parameter $A$ from equations (25) and (26) leads to the quadratic equation for the parameter $B$ as

$$
20 c v B^{2}+b_{1}-b_{2} v=0
$$

which leads to

$$
B=\frac{1}{2} \sqrt{\frac{b_{2} v-b_{1}}{5 c v}} .
$$

Finally, equating the two values of the parameter $B$ from equations (24) and (28) leads to the quadratic equation for the speed $v$ of the shock wave as

$$
\left(3 b_{2}^{2}-50 c\right) v^{2}+\left(50 a c-6 b_{1} b_{2}\right) v+3 b_{1}^{2}=0
$$

whose solution is

$$
v=\frac{3 b_{1} b_{2}-25 a c+5 \sqrt{25 a^{2} c^{2}+6 b_{1} c\left(b_{1}-a b_{2}\right)}}{3 b_{2}^{2}-50 c}
$$

provided

$$
3 b_{2}^{2} \neq 50 c
$$

and

$$
25 a^{2} c^{2}+6 b_{1} c\left(b_{1}-a b_{2}\right)>0 .
$$

This expression for the speed of the shock wave is in terms of the known given parameters that is retrievable only using this approach.

The following figure shows the profile of a shock wave for $p=1, a=b_{1}=1, b_{2}=-1, c=k=1, A=1$. 


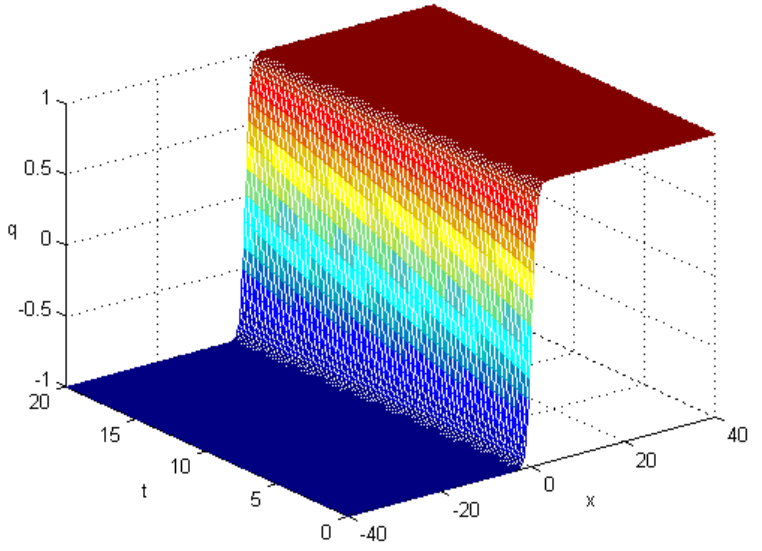

Figure 2: Shock waves with $p=1, a=1, b 1=1, b 2=-1, c=1, k=1, A=1$.

\subsubsection{Case-II: $\mathrm{p}=2$}

For $p=2, n=3$ by virtue of equation (6). In this case, equation (1) reduces to

$$
q_{t}+a q_{x}+b_{1} q_{x x x}+b_{2} q_{x x t}+c q_{x x x x}+k\left(q^{3}\right)_{x}=0 .
$$

Similarly, as in the case for $p=1$, the speed of the shock wave is given by

$$
v=\frac{a-8 b_{1} B^{2}}{136 c B^{4}-8 b_{2} B^{2}+1}
$$

and

$$
v=\frac{a-20 b_{1} B^{2}}{616 c B^{4}-20 b_{2} B^{2}+1} .
$$

Upon equating the two expressions for the speed $v$ given by (34) and (35), leads to the biquadratic equation for the parameter $B$ as

$$
184 b_{1} c B^{4}-40 a c B^{2}-\left(b_{1}-a b_{2}\right)=0
$$

whose solution is

$$
B=\left[\frac{10 a c-\sqrt{100 a^{2} c^{2}+46 b_{1} c\left(b_{1}-a b_{2}\right)}}{92 b_{1} c}\right]^{\frac{1}{2}} .
$$

Again from the coefficients of the linearly independent functions $\tanh ^{p+j} \tau$, for $j=3,5$ gives the free parameter $A$ as

$$
A=2 B^{2}\left(\frac{30 c v}{k}\right)^{\frac{1}{2}}
$$

and

$$
A=2 B\left[\frac{\left(b_{1}-b_{2} v+70 v c B^{2}\right)}{k}\right]^{\frac{1}{2}}
$$

Equating the two expressions for the parameter $A$ from equations (38) and (39) leads to the quadratic equation for the second parameter $B$ of the shock wave given by

$$
40 c v B^{2}+b_{1}-b_{2} v=0
$$

that yields

$$
B=\frac{1}{2} \sqrt{\frac{b_{2} v-b_{1}}{10 c v}} .
$$

Next, equating the two values of the parameter $B$ from equations (37) and (41) gives a quadratic equation for the speed $v$ of the shock wave as

$$
\left(23 b_{2}^{2}-200 c\right) v^{2}+\left(200 a c-46 b_{1} b_{2}\right) v+23 b_{1}^{2}=0
$$

which solves to

$$
v=\frac{23 b_{1} b_{2}-100 a c+10 \sqrt{100 a^{2} c^{2}+46 b_{1} c\left(b_{1}-a b_{2}\right)}}{23 b_{2}^{2}-200 c}
$$

as long as

$$
23 b_{2}^{2} \neq 200 c
$$

and

$$
100 a^{2} c^{2}+46 b_{1} c\left(b_{1}-a b_{2}\right)>0 .
$$

This is the expression for the speed of the shock wave, for $n=3$, in terms of the given parameters as long as the constraint connections between the known parameters, given by equations (44) and (45), hold.

The following figure shows the profile of a shock wave for $p=2, a=b_{1}=1, b_{2}=-1, c=k=1, A=1$.

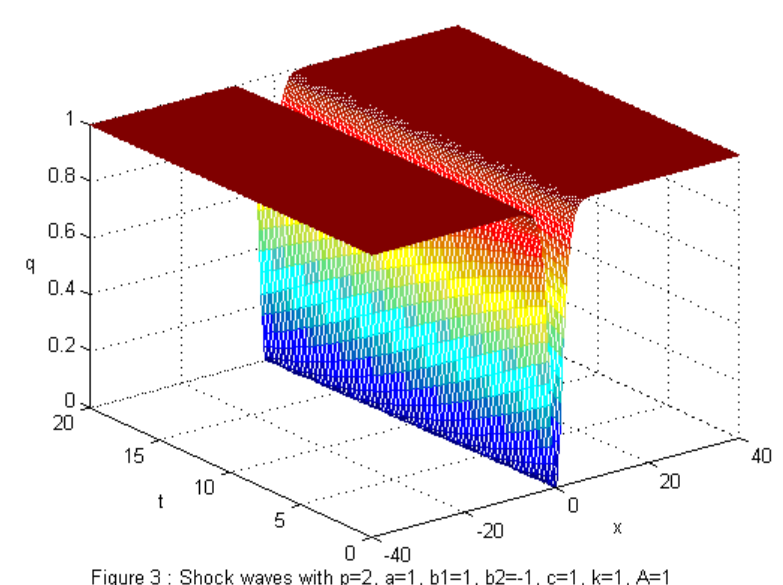

2.3 Singular Solitons

This subsection will retrieve the singular soliton solution to the R-KdV-RLW equation. Singular solitons are 
unwanted features in any nonlinear evolution equations. These solutions are spikes and therefore can possibly provide an explanation to the formation of Rogue waves, provided the conditions are just right. The starting hypothesis is given by [15]

$$
q(x, t)=A \operatorname{csch}^{p} \tau
$$

where $A$ and $B$ are free parameters and $\tau$ is still defined as in equation (4). Substituting equation (46) into equation (1) leads to

$$
\begin{gathered}
\left\{v\left(1+b_{2} p^{2} B^{2}+c p^{4} B^{4}\right)-a-b_{1} p^{2} B^{2}\right\} \operatorname{csch}^{p} \tau \\
-B^{2}(p+1)(p+2)\left\{b_{1}-b_{2} v-2 c v B^{2}\left(p^{2}+2 p+2\right)\right\} \operatorname{csch}^{p+2} \tau \\
+c v B^{4}(p+1)(p+2)(p+3)(p+4) \operatorname{csch}^{p+4} \tau \\
-n k A^{n-1} \operatorname{csch}^{n p} \tau=0
\end{gathered}
$$

and then proceeding as in subsection 2.1 gives the singular soliton soliton solution as

$$
q(x, t)=A \operatorname{csch}^{\frac{4}{n-1}}[B(x-v t)] .
$$

with the same definition of the parameters as well as the same constraint conditions as in Section 2.1.

\section{Conservation Laws}

There are two conserved quantities for the R-KdV-RLW equation with power law nonlinearity. They are the momentum $(M)$ and the energy $(E)$. These are respectively given by

$$
M=\int_{-\infty}^{\infty} q d x=\frac{A}{B} \frac{\Gamma\left(\frac{1}{2}\right) \Gamma\left(\frac{2}{n-1}\right)}{\Gamma\left(\frac{1}{2}+\frac{2}{n-1}\right)}
$$

and

$$
\begin{aligned}
& E=\int_{-\infty}^{\infty}\left\{q^{2}-b_{2}\left(q_{x}\right)^{2}+c\left(q_{x x}\right)^{2}\right\} d x \\
&=A^{2}\left[\left\{(n-1)^{2}(n+7)(3 n+5)-\right.\right. 16 b_{2}(n-1)(3 n+5) B^{2} \\
&\left.\left.+256(n+2) c B^{4}\right\} /\left\{B(n-1)^{2}(n+7)(3 n+5)\right\}\right] \times \frac{\Gamma\left(\frac{1}{2}\right) \Gamma\left(\frac{4}{n-1}\right)}{\Gamma\left(\frac{1}{2}+\frac{4}{n-1}\right)}
\end{aligned}
$$

The 1-soliton solution given by (15) is used to compute these conserved quantities from their densities.

\section{Semi-inverse Variational Principle}

This section integrates the R-KdV-RLW equation by the semi-inverse variational principle (SVP) that is also known as the inverse problem approach. This approach will lead to an analytical 1-soliton solution to the governing equation (1) that is not an exact solution. There are constraint conditions that need to hold in this case as well. They will naturally fall out during the course of derivation of the solution. Therefore, in order to solve equation (1), the starting hypothesis is taken to be $[4,8,11]$

$$
q(x, t)=g(x-v t)=g(s)
$$

where

$$
s=x-v t
$$

and $v$ is the speed of the solitary wave while $g$ represents the wave profile. Substituting (51) into (1) and integrating once with respect to $s$ gives

$$
(v-a) g-\left(b_{1}-b_{2} v\right) g^{\prime \prime}+c v g^{\prime \prime \prime \prime}-k\left(g^{n}\right)=0
$$

where $g^{\prime \prime}=d^{2} g / d s^{2}$ and $g^{\prime \prime \prime \prime}=d^{4} g / d s^{4}$ and the integration constant is taken to be zero, without any loss of generality. Now, multiplying both sides of equation (53) by $g^{\prime}$ and integrating once more, leads to

$$
\begin{aligned}
(v-a) g^{2}- & \left(b_{1}-b_{2} v\right)\left(g^{\prime}\right)^{2} \\
& +c v\left\{2 g^{\prime} g^{\prime \prime \prime}-\left(g^{\prime \prime}\right)^{2}\right\}-\frac{2 k g^{n+1}}{n+1}=K,
\end{aligned}
$$

where $K$ is the integration constant. The stationary integral is then defined as $[4,8,11]$

$$
\begin{aligned}
J=\int_{-\infty}^{\infty} K d s & =\int_{-\infty}^{\infty}\left[(v-a) g^{2}-\left(b_{1}-b_{2} v\right)\left(g^{\prime}\right)^{2}\right. \\
& \left.+c v\left\{2 g^{\prime} g^{\prime \prime \prime}-\left(g^{\prime \prime}\right)^{2}\right\}-\frac{2 k g^{n+1}}{n+1}\right] d s
\end{aligned}
$$

The hypothesis for 1-soliton solution to (1) is taken to be

$$
g(s)=A \operatorname{sech}^{\frac{4}{n-1}}[B(x-v t)] .
$$

Substituting this hypothesis into (55) and carrying out the integrations, leads to

$$
\begin{gathered}
J=\left[\frac{(v-a) A^{2}}{B}-\frac{16 b A^{2} B}{(n-1)(n+7)}-\frac{768(n+2) c v A^{2} B^{3}}{(n-1)^{2}(n+7)(3 n+5)}\right. \\
\left.-\frac{32(n+3) k A^{n+1}}{(n+1)(n+7)(3 n+5) B}\right] \frac{\Gamma\left(\frac{4}{n-1}\right) \Gamma\left(\frac{1}{2}\right)}{\Gamma\left(\frac{4}{n-1}+\frac{1}{2}\right)} .
\end{gathered}
$$

Then, SVP states that the amplitude $(A)$ and the width $(B)$ of the soliton can be obtained after solving the coupled syetem of equations given by $[4,8,11]$

$$
\frac{\partial J}{\partial A}=0
$$

and

$$
\frac{\partial J}{\partial B}=0
$$


These lead to

$$
\begin{aligned}
v-a-\frac{16\left(b_{1}-b_{2} v\right) B^{2}}{(n-1)(n+7)}- & \frac{768(n+2) c v B^{4}}{(n-1)^{2}(n+7)(3 n+5)} \\
& -\frac{16 k(n+3) A^{n-1}}{(n+7)(3 n+5)}=0
\end{aligned}
$$

and

$$
\begin{array}{r}
v-a+\frac{16\left(b_{1}-b_{2} v\right) B^{2}}{(n-1)(n+7)}+\frac{2304(n+2) c v B^{4}}{(n-1)^{2}(n+7)(3 n+5)} \\
-\frac{32 k(n+3) A^{n-1}}{(n+1)(n+7)(3 n+5)}=0 .
\end{array}
$$

Eliminating the amplitude $A$ between equations (60) and (61) leads to the biquadratic equation for the width $B$ as

$$
\begin{aligned}
256 P_{2} B^{4}+32(n-1)(n & +7)\left(b_{1}-b_{2} v\right) B^{2} \\
& +P_{1}(n-1)^{2}(n+7)^{2}=0
\end{aligned}
$$

where

$$
P_{1}=\frac{16 k(n-1)(n+3) A^{n-1}}{(n+1)(n+7)(3 n+5)}
$$

and

$$
P_{2}=\frac{12(n+2)(n+7) c v}{3 n+5} .
$$

Therefore the solution to (62) is

$$
B=\frac{1}{4}\left[\frac{(n-1)(n+7)\left\{-\left(b_{1}-b_{2} v\right)+\sqrt{\left(b_{1}-b_{2} v\right)^{2}+P_{1} P_{2}}\right\}}{P_{2}}\right]_{(65)}^{\frac{1}{2}}
$$

for

$$
\left(b_{1}-b_{2} v\right)^{2}+P_{1} P_{2}>0
$$

and

$$
P_{2}\left\{-\left(b_{1}-b_{2} v\right)+\sqrt{\left(b_{1}-b_{2} v\right)^{2}+P_{1} P_{2}}\right\}>0 .
$$

Then the amplitude $A$ can be computed by substituting the width $B$ into (60) or (61). The velocity of the soliton is given by (60) or (61).

\section{Conclusion}

This paper addressed the dynamics of shallow water waves by the R-KdV-RLW equation with power law nonlinearity. Additionally, shock wave solutions and the singular soliton solutions are retrieved. Finally, the SVP is utilized to retrieve a single solitary wave solution although this is not an exact solution, yet analytical.

The results of this manuscript is going to be profoundly helpful towards further ongoing research on shallow water waves. The perturbation terms will be added and the soliton perturbation theory will be implemented to obtain the adiabatic parameter dynamics of the solitary waves. With strong perturbations, the extended R-KdV-RLW equation will be integrated for exact as well as other analytical solitons. There will be several integration architectures that will be implemented into retrieving the solutions. Later, the stochastic perturbation terms will be taken into account and the mean free velocity of the solitons will be obtained after solving the corresponding the Langevin equation. These just form a tip of the iceberg.

\section{References}

[1] S. M. Abbas, Ilyas Saleem, Bilal Ahmed, Hunaina Khurshid, UWB Antenna with Parasitic Patch and Asymmetric Feed, Information Science Letters, 2, 27-33 (2013).

[2] M. Antonova \& A. Biswas. Adiabatic parameter dynamics of perturbed solitary waves. Communications in Nonlinear Science and Numerical Simulation. 14, 734-748 (2009).

[3] A. Biswas, H. Triki \& M. Labidi. Bright and dark solitons of the Rosenau-Kawahara equation with power law nonlinearity. Physics of Wave Phenomena. 19, 24-29 (2011).

[4] A. Biswas. Soliton solutions of the resonant nonlinear Schrödinger's equation with full nonlinearity by semi-inverse variational principle. Quantum Physics Letters. 1, 79-84 (2012).

[5] G. Ebadi, A. Mojaver, H. Triki, A. Yildirim \& A. Biswas. Topological solitons and other solutions of the Rosenau-KdV equation with power law nonlinearity. Romanian Journal of Physics. 58, 3-14 (2013).

[6] A. Esfahani. Solitary wave solutions for generalized Rosenau-KdV equation. Communications in Theoretical Physics. 55, 396-398 (2011).

[7] L. Girgis \& A. Biswas. Soliton perturbation theory for nonlinear wave equations. Applied Mathematics and Computation. 216, 2226-2231 (2010).

[8] L. Girgis \& A. Biswas. A study of solitary waves by He's variational principle. Waves in Random and Complex Media. 21, 96-104. (2011).

[9] Awatif S. Jassim, Rasha A. Abdullah, Faleh L. Matar and Mohammed A. Razooqi, The Effect of Photo irradiation by Low Energy Laser on the Optical Properties of Amorphous GaAs Films, International Journal of Thin Films Science and Technology, 1, 9-13 (2012).

[10] Bo Zhang and Zhicai Juan, Modeling User Equilibrium and the Day-to-day Traffic Evolution based on Cumulative Prospect Theory, Information Sciences Letters, 2, 9-12 (2013).

[11] M. Labidi \& A. Biswas. Application of He's principles to Rosenau-Kawahara equation. Mathematics in Engineering, Science and Aerospace. 2, 183-197 (2011).

[12] D. A. Lott, A. Henriquez, B. J. M. Sturdevant \& A. Biswas. Optical soliton-like structures resulting from the nonlinear Schrödinger's equation with saturable law nonlinearity. Applied Mathematics and Information Sciences. 5, 1-16 (2011).

[13] X. Pan \& L. Zhang. Numerical simulation for general Rosenau-RLW equation: An average linearized conservative 
scheme. Mathematical Problems in Engineering. 2012, 517818 (2012).

[14] Akoteyon, I.S, Evaluation of Groundwater Quality Using Contamination Index in Parts of ALIMOSHO, LAGOSNIGERIA, American Academic \& Scholarly Research Journal, 4, (2012).

[15] P. Razborova, H. Triki \& A. Biswas. Perturbation of dispersive shallow water waves. Ocean Engineering. 63, 1-7 (2013).

[16] Tamer Shahwan and Raed Said, A Comparison of Bayesian Methods and Artificial Neural Networks for Forecasting Chaotic Financial Time Series, Journal of Statistics Applications \& Probability, 1, 89-100 (2012).

[17] M. Wang, D. Li \& P. Cui. A conservative finite difference scheme for the generalized Rosenau equation. International Journal of Pure and Applied Mathematics. 71, 539-549 (2011).

[18] Muhammad Shuaib Khan and Robert King, Modified Inverse Weibull Distribution, Journal of Statistics Applications \& Probability, 1, 115-132 (2012).

[19] J-M. Zuo. Solitons and periodic solutions for the Rosenau-KdV and Rosenau-Kawahara equations. Applied Mathematics and Computation. 215, 835-840 (2009).

[20] J-M. Zuo, Y-M. Zhang, T-D. Zhang \& F. Chang. A new conservative difference scheme for the generalized RosenauRLW equation. Boundary Value Problems. 2010, Article 516260, (2010).

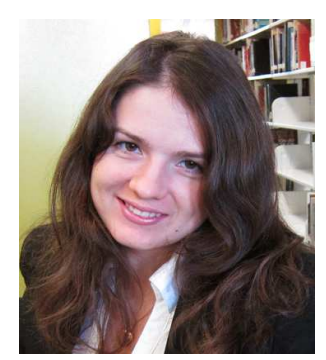

Polina Razborova, originally from Russia, is a graduate student in the Department of Mathematical Sciences at Delaware State University (DSU). She earned her BS degree from DSU with a major in Management and minor in Mathematics. Subsequently, she earned her MS degree in Applied Mathematics, also from DSU. Currently, she is pursuing her doctoral studies at DSU. Her research interest is in Theory of Solitons.

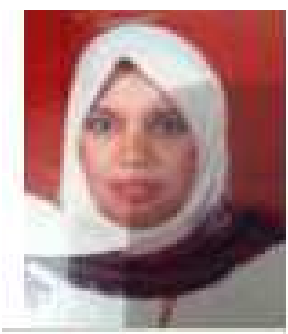

\section{Bouthina}

Ahmed, Doctor of Numerical Analysis in Mathematics Department Women Science - Ain Shams University, Cairo, Egypt. Her MS degree is in differential equations and Ph.D degree is in numerical analysis. Her research area of interest is applied mathematics.

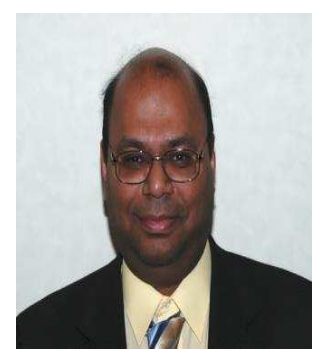

Anjan Biswas earned his BS degree in Mathematics from St. Xavier's College in Calcutta, India. Subsequently he obtained his M.Sc and M.Phil degrees in Applied Mathematics from the University of Calcutta, India. After that, he earned his MA and Ph.D. degrees in Applied Mathematics from the University of New Mexico in Albuquerque, NM, USA. Currently, he is an Associate Professor of Mathematics in the Department of Mathematical Sciences at Delaware State University in Dover, DE, USA. His current research area of interest is Theory of Solitons. 